\title{
Neural Network Based Model for a PEM Fuel Cell System
}

\author{
I. Zamora ${ }^{1}$, J.I. San Martín ${ }^{2}$, J.J. San Martín ${ }^{2}$, V. Aperribay ${ }^{2}$, P. Eguía ${ }^{1}$ \\ Department of Electrical Engineering - University of the Basque Country \\ ${ }^{1}$ Escuela Técnica Superior de Ingeniería de Bilbao \\ Alda. Urquijo s/n, 48013 Bilbao (Spain) - e_mail: inmaculada.zamora@ehu.es \\ ${ }^{2}$ Escuela Universitaria de Ingeniería Técnica Industrial de Eibar \\ Avda. Otaola, 29, 20600 Eibar (Spain) - e_mail: iepsadij@sb.ehu.es
}

\begin{abstract}
Usually, modelling of fuel cell systems uses complex expressions, based on the knowledge of physicalchemical phenomena. These models require a good knowledge of the parameters involved in the processes that, in many cases, are difficult to determine. A solution to avoid this difficulty consists in using black-box models, such as those based on artificial neural networks (ANN).

This paper presents the modelling of a PEM fuel cell system, using ANNs. The selected ANN structure has been validated for the transient state, during the start up of the system, and in steady state. Results are shown for a commercial PEM fuel cell system.
\end{abstract}

\section{Keywords}

Fuel Cells, PEMFC, System Identification, Modelling, ANN.

\section{Introduction}

Fuel cells are highly non-linear systems. So, appropriate system identification techniques should be used to obtain, in an experimental way, a good model. This model should reproduce, with enough accuracy, the dynamic characteristics of the analysed process.

Currently, a high number of models that describe the behaviour of a fuel cell exist, estimating the voltage for a given group of parameters. However, few models are able to predict the transition process between two steady state points. In this sense, around $80 \%$ of the operating situations in fuel cells applied to road transport is dynamic [1]. For that reason, dynamic simulations are needed to improve the reliability of fuel cell systems. However, the models based on physical principles, described by means of differential equations, are quite complex and need a high computing time.

Figure 1 shows a classification of different existent models to characterize the lineal and non-linear systems, with static and dynamic characteristics [2].

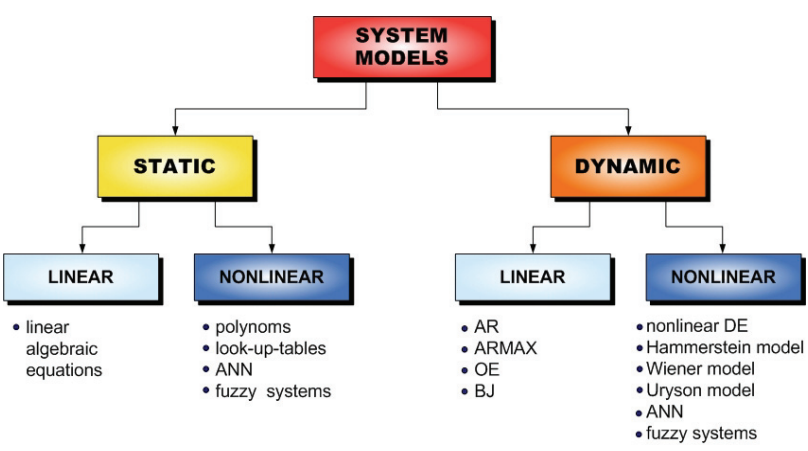

Fig. 1. Models classification for static and dynamic systems

In this context, this paper presents the modelling of the behaviour of a fuel cell system by means of ANNs. This technique needs to know the inputs and its corresponding outputs, without the need of additional information. Data required for the model have been obtained from measurements taken in a commercial PEM fuel cell system.

\section{General aspects of PEM fuel cells}

Fuel cells can be classified based on two fundamental aspects:

- Operating temperature (low or high).

- Electrolyte material (substance used as a bridge to exchange ions between the anode and cathode).

Regarding the temperature, four models (AFC, PEMFC, DMFC, PAFC) are considered of low temperature and two models (MCFC y SOFC) of high temperature.

PEM fuel cells work at $80^{\circ} \mathrm{C}$ and the electrolyte consists of a solid polymer layer, usually Nafion (based on a polyethylene polymer). The anode, or fuel electrode, is $\mathrm{Pt} / \mathrm{C}$ deposited on coal paper and the cathode, or air electrode, is also $\mathrm{Pt} / \mathrm{C}$. 
They are devices where chemical reaction energy becomes in electricity without combustion. Their high efficiency, silent character, low emissions of pollutants and fuel flexibility can be highlighted. They can use hydrogen as fuel, being transformed directly in electrical energy in a similar process to conventional batteries. Hydrogen appears being part of other fuels, like natural gas, methane, propane, ethanol, etc., where it can be extracted of to be used in the cell. Hydrogen production with renewable sources, and its later use in fuel cells, facilitates a clean energy source to which an interesting future is expected.

Fuel cells do not depend on the Carnot cycle, so that, ideally, the free energy of the reaction can be converted totally into electricity. In practise, nevertheless, electrodes over potentials produce efficiencies lower than $100 \%$. It must be observed that electrochemical reactions produced are exothermic, so that the combined use of electricity and heat (cogeneration) produces a higher net efficiency.

When the electrolyte is solid, they useful life of the fuel cell is long, around 50,000 hours. They can supply rated output after three minutes of operation. Every cell supplies around $0.7 \mathrm{Vdc}$ with current densities close to $900 \mathrm{~mA} / \mathrm{cm}^{2}$. "Air bleed" technique allows this kind of cells to operate with hydrogen obtained from alcohols or carbonated fuels.

\section{PEMFC modelling with ANNs}

The empiric equations used to model a PEMFC stack, give the fuel cell voltage as indicated in expressions (1) and (2), [3], [4].

$$
\begin{gathered}
E=E_{o}-b \cdot \log i-R \cdot i-m \cdot \exp (n i) \\
E=E_{o}-b \cdot \log i-R \cdot i-\alpha \cdot i^{K} \cdot \operatorname{Ln}(1-\beta i)
\end{gathered}
$$

Where: $E_{o}$ is the open circuit voltage; $b$ and $R$ represent the Tafel slope and the ohmic resistance, respectively, and $m, n, \alpha, \beta$ and $K$ are constants of the empiric equation. These parameters are derived from mathematical and statistical considerations.

The problem is that the value of the aforementioned constants depends on the physical properties and the operating conditions of the fuel cell. On the other hand, the mathematical relationship between these constants and the physical properties or operating conditions is not well defined [5].

In [6] and [7], these constants are presented, under representative operating conditions. However, to avoid the calculation of those constants in a wide range of operating conditions, techniques based on ANNs can be used to model the physical processes [8].

ANNs simulate the behaviour of natural systems by means of the interconnection of basic processing units called neurons. Neurons are highly connected with each other by means of links. They can receive external signals or signals coming from other neurons affected by a factor called 'weight'. The output of the neuron is the result of applying a specific function, known as 'activation or transfer function', to the sum of its inputs, plus a threshold value called 'bias'. With these general characteristics it is possible to develop different network structures.

Among the most important topologies of ANNs, we can highlight the following:

- Multi-Layer-Perceptron with the learning rule Backpropagation.

- Competitive Neural Network or Kohonen Neural Network.

- Hopfield Neural Network.

The ANN topology most used is the Multilayer Perceptron type (MLP), in which the neurons are organized in layers, [9], [10]. In this topology, the outputs of a layer are the inputs of the following one. The layers located among the input and output layers are called hidden layers. Figure 2 shows an example of this ANN topology, constituted by the input layer, two hidden layers and the output layer.

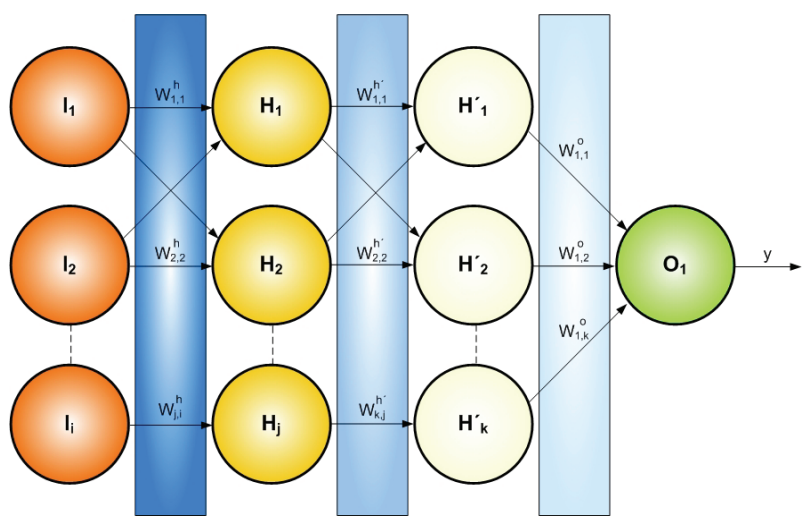

Fig. 2. Example of ANN structure

Where:

$I_{i}$ : $\quad$ Input neurons

$H_{j}, H_{k}{ }^{\prime}$ : Hidden neurons

$O$ : Output neuron

$W_{j, i}^{h}$ : Weight matrix element between the hidden neuron $j$ and the input $i$

$W_{k, j}^{h^{\prime}}$ : Weight matrix element between the hidden neuron $j$ and the hidden neuron $k$

$W_{m, k}^{o}$ : Weight matrix element between the hidden neuron $k$ and the output $m$

Besides, the back-propagation learning rule is used in perhaps 80 to $90 \%$ of practical applications. The backpropagation learning rule is used to adjust the weights and biases of the network in order to minimise the sumsquared error of the network. This is done by continually changing the values of the network weights and biases in 
the direction of steepest decrement with respect to the error. As the simple back-propagation method is slow, because it requires small learning rates for stable learning, improvement techniques such as momentum and adaptive learning rate or an alternative method to gradient descent, Levenberg-Marquardt optimisation, can be used.

To implement a neural network, the following steps must be taken:

- Select a suitable network architecture.

- Select the learning rule best suited to the chosen network.

- Train the neural network.

- Validate the network behaviour.

Depending on the type of problem we are dealing with and its specific conditions, the most adequate network structure will be different. Besides, within the same type of neural network, there are several configurations: number of layers, number of neurons in each layer, transfer functions and learning rules.

To train the network, a suitable number of representative examples must be selected. This way, the network can learn the fundamental characteristics of the problem so that, once the training process is completed, right results can be obtained corresponding to new situations not considered during training.

After the training process and therefore, after the determination of the weights and biases of each neuron of the network, the results of the training process are validated, studying how the network performs under different conditions that may not have been considered in the training process.

For the modelling of the PEM fuel cell presented in this paper, both training and validation have been developed using the software tool SARENEUR [11].

\section{Application of SARENEUR}

Although the modelling using ANNs has great advantages, also presents some disadvantages. Among them, the complexity in selecting the right type of network and its architecture (number of layers, number of neurons per layer, activation functions, learning algorithm parameters, etc) can be emphasized. In fact, the number of structures that can be used for solving a problem is very large, and so, it is necessary to make a good selection of the network to use. For this purpose a software tool, called SARENEUR [11], has been used. This software has been developed in the Matlab environment and allows selecting and verifying the performance of neuronal networks.

SARENEUR is a software package of general application that allows determining the proper ANN structure to any type of problem for which the solution using neural networks can be applied. This tool is structured in three modules linked as shown in figure 3 .

- Input interface module, which aims at obtaining, processing and preparing the necessary data for training and checking the network.

- Verification and selection module, which generates the different networks and checks their performance.

- Results display module, which shows the results provided by the verification and selection module.

\section{SARENEUR}

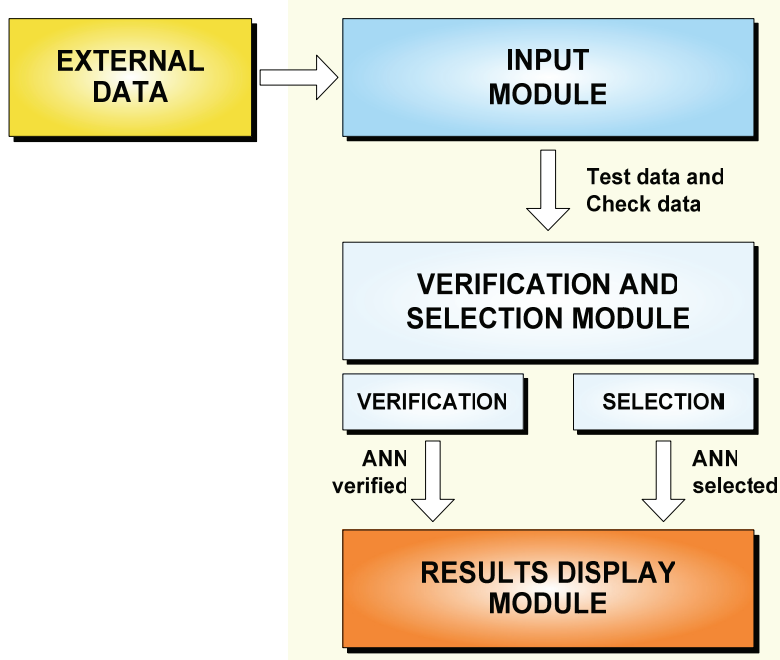

Fig. 3. SARENEUR flowchart diagram

First of all, the type of ANN and the learning rule must be defined. For our model, the Multi-Layer-Perceptron with the Backpropagation learning rule has been selected.

Besides, one factor in determining the right size and structure for the network is the number of inputs and outputs which it must have. The lower the number of inputs, the smaller the network can be. However, sufficient input data to characterize the problem must be met. Thus, the following parameters have been considered:

- Input parameters: fuel cell temperature, hydrogen consumption, hydrogen pressure, oxygen flow and inlet air temperature.

- Output parameters: fuel cell voltage and current.

After deciding the inputs and outputs of the network, the number of layers and the number of neurons per layer has to be considered. This information is obtained using the SARENEUR tool. Thus, once the matrix of input and output data have been constructed, this tool allows developing an iterative process, considering the following ranges in the training parameters (see figure 4):

$-1^{\text {st }}$ Hidden layer: from 1 to 11 neurons

$-2^{\text {nd }}$ Hidden layer: from 0 to 6 neurons 
- Transfer functions: possible combinations with logsigmoid and tan-sigmoid functions in the hidden layers and linear functions in the output layer

- Learning rule: Levenberg-Marquardt

- Learning parameters:

- Min. Performance gradient: 1e-10

- $\operatorname{Max} \mu: 1 \mathrm{e} 10$

- Max. Validation failures: 5

- Training options:

- Error: 1e-5

- Max. Time: $180 \mathrm{~s}$

- Max. Number of iterations: 500

Parameters to select the right ANN structures:

- Type of error: relative

- Average error: 0.02

- Max. Allowable error: 0.03

- Max. Desired error: 0.02

- $\%$ of cases with error above the desired error: $1 \%$

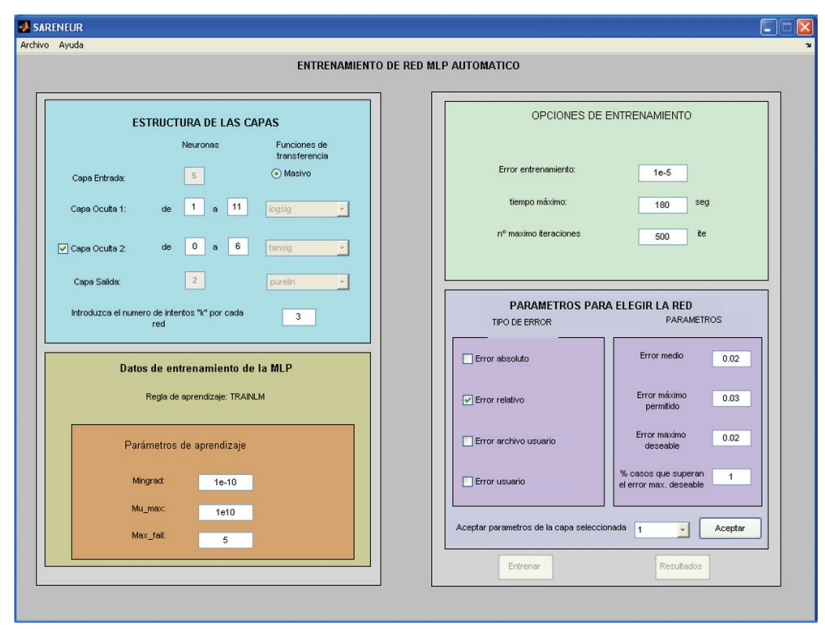

Fig. 4. Parameters selection with SARENEUR

When applying the SARENEUR tool, two different situations have been considered: the fuel cell in steady state and the start up transient. Initially, several valid structures have been obtained for the steady state, and their performance have been validated with data measured during the start up transient. Afterwards, a solution has been proposed that uses the same ANN structure for both situations, with good agreement with the measurements.

\section{A. Steady state}

To model the PEM fuel cell, described in section 5, in steady state, the data have been collected through measurements in the laboratory. Multiple tests have been developed for the training process and 100 samples have been considered for each of the five inputs and two outputs. Using SARENEUR, several ANN structures that comply with the requirements stated have been identified. These structures are shown in Table I.
Table I - The selected ANN structures

\begin{tabular}{|c|c|c|c|c|c|}
\hline $\begin{array}{l}\text { TRANSFER } \\
\text { FUNCTION }\end{array}$ & $\begin{array}{c}\text { NEURONS } \\
\text { 1st hidden } \\
\text { layer }\end{array}$ & $\begin{array}{l}\text { NEURONS } \\
\text { 2nd hidden } \\
\text { layer }\end{array}$ & TIME & $\begin{array}{l}\text { AVERAGE } \\
\text { ERROR }\end{array}$ & $\begin{array}{c}\text { MAXIMUM } \\
\text { ERROR }\end{array}$ \\
\hline LOGSIG-PURELIN & 4 & 0 & 2,52 & 0,001 & 0,005 \\
\hline LOGSIG-PURELIN & 4 & 0 & 2,34 & 0,001 & 0,008 \\
\hline LOGSIG-PURELIN & 5 & 0 & 3,08 & 0,002 & 0,018 \\
\hline LOGSIG-PURELIN & 8 & 0 & 3,04 & 0,001 & 0,012 \\
\hline $\begin{array}{c}\text { TANSIG-LOGSIG- } \\
\text { PURELIN }\end{array}$ & 10 & 6 & 6,81 & 0,000 & 0,008 \\
\hline TANSIG-PURELIN & 7 & 0 & 2,88 & 0,001 & 0,013 \\
\hline TANSIG-PURELIN & 11 & 0 & 3,84 & 0,001 & 0,007 \\
\hline TANSIG-PURELIN & 10 & 0 & 3,57 & 0,000 & 0,014 \\
\hline $\begin{array}{l}\text { TANSIG-TANSIG- } \\
\text { PURELIN }\end{array}$ & 10 & 6 & 6,56 & 0,001 & 0,005 \\
\hline
\end{tabular}

\section{B. Start up transient}

A test has been carried out to check if the ANN structures obtained for operation in steady state work properly during the start up transient. Available data of each variable, obtained during the start up of the fuel cell, have been used.

Figures 5 and 6 compare the results for the two ANN structures of table I, which converge with the measured values in the start up transient. It is clear that the output of the networks do not agree with the measurements. The main differences appear in the beginning of the transient, especially in the voltage curve.

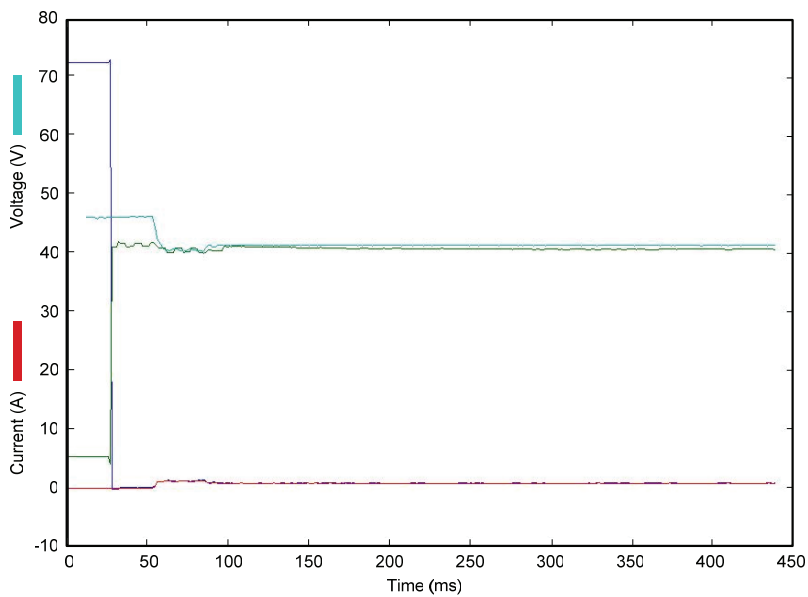

Fig. 5. ANN 5-5-0-2_Logsig-Purelin

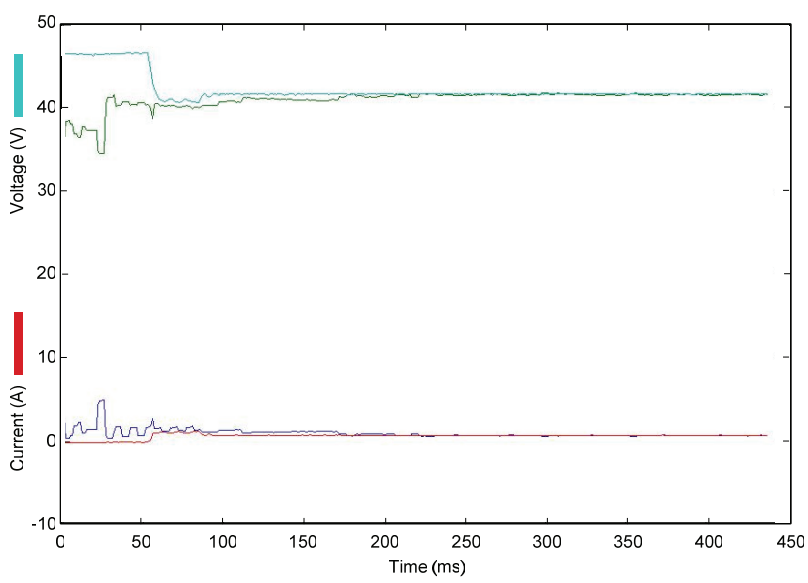

Fig. 6. ANN 5-10-6-2_Tansig-Tansig-Purelin 


\section{Steady state + Start up transient}

The range of values of several input parameters to the ANN model of the fuel cell can be quite different during the start up and the steady state. For this reason, it is difficult to use the same ANN structure to model both operating states. With the aim of obtaining only one structure of ANN, which can be applied in both situations, it was decided to apply a logarithmic scale to the input values, as shown in expression (3).

$$
\text { new data }=\log (\text { old data }+10)
$$

With this new scale applied to the input data, the two ANN structures previously selected have a good performance in both situations, as shown in figures 7 and 8 .

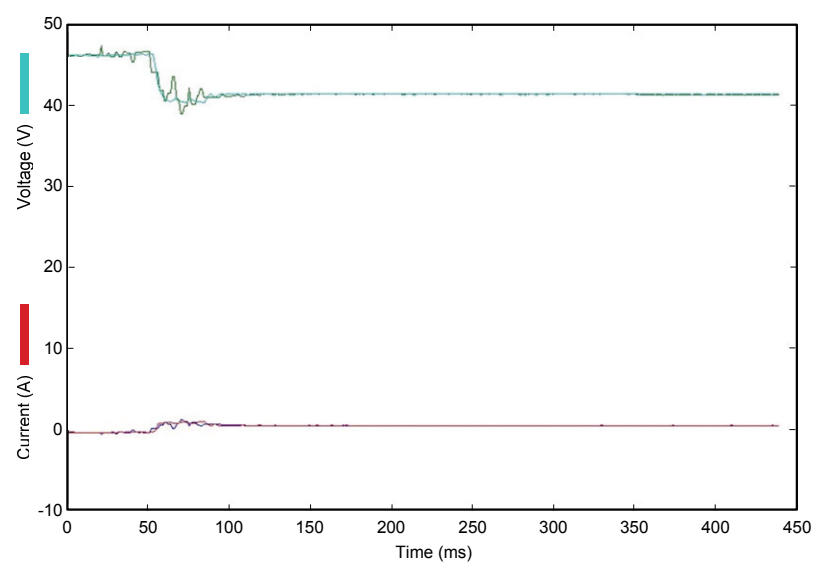

Fig. 7. ANN 5-5-0-2_Logsig-Purelin (logarithmic scale)

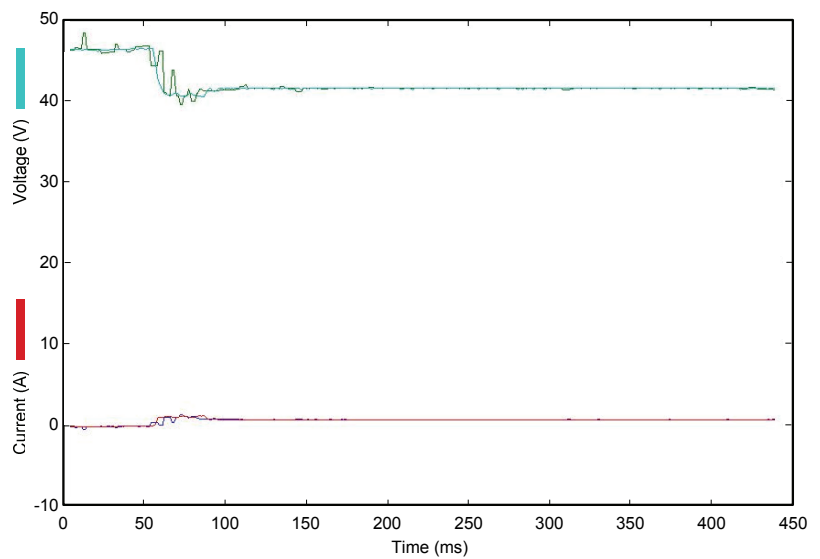

Fig. 8. ANN 5-10-6-2_Tansig-Tansig-Purelin (logarithmic scale)

\section{Results}

A group of experimental measurements have been made of a commercial Fuel Cell System Nexa Ballard of $1.2 \mathrm{~kW}$ to obtain enough data to train and test the selected ANN structure. These measurements include different parameters, such as: temperature of the stack of the fuel cell system, current, voltage, hydrogen consumption, hydrogen pressure, air flow stoichiometry, etc. For each parameter, a total of 2004 samples have been recorded.
The Nexa Power Module (Ballard Power Systems Inc.) is a fuel cell system that needs low maintenance and is completely automated. This system has been designed to be integrated in portable products and in back-up power markets. Figure 9 details the location of the fuel cell in the test system layout used in the laboratory. Its technical specifications are included in figure 10. Finally, figure 11 shows a screen of the software that allows taking measures from the fuel cell.

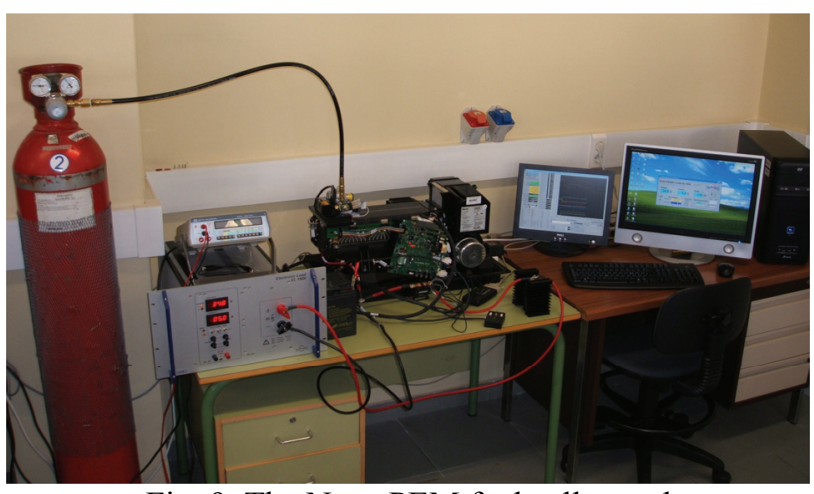

Fig. 9. The Nexa PEM fuel cell tested

\begin{tabular}{|c|c|c|}
\hline FUEL CELL & \multicolumn{2}{|c|}{ NEXA $^{\mathrm{TM}}$} \\
\hline \multirow{5}{*}{ PERFORMANCE } & Rated net output power & $1200 \mathrm{~W}$ \\
\hline & Heat disipation & $1600 \mathrm{~W}$ (at rated net output) \\
\hline & Current & 46 A CC (at rated net output) \\
\hline & Voltage & 26 V CC (at rated net output) \\
\hline & Lifetime & 1500 hours \\
\hline \multirow{2}{*}{ FUEL } & Gaseus hydrogen & $99,99 \%$, dry \\
\hline & Supply pressure & 10 to 250 PSIG \\
\hline \multirow{2}{*}{$\begin{array}{l}\text { OPERATING } \\
\text { ENVIRONMENT }\end{array}$} & Ambient temperature & 3 to $40^{\circ} \mathrm{C}$ \\
\hline & Humidity & 0 a $95 \%$ non-condensing \\
\hline \multirow{3}{*}{ EMISSIONS } & Pure water (vapor anc liquid) & Max $.870 \mathrm{~m} / \mathrm{h}$ (at rated net output) \\
\hline & $\mathrm{CO}, \mathrm{CO}_{2}, \mathrm{NO}_{x}, \mathrm{SO}_{2}$ particulates & $0 \mathrm{ppm}$ \\
\hline & Noise & 72 dBA @ 1m \\
\hline \multirow{2}{*}{ PHYSICAL } & Dimensions & $56 \times 25 \times 33 \mathrm{~cm}$ \\
\hline & Weight & $13 \mathrm{~kg}$ \\
\hline
\end{tabular}

Fig. 10. Nexa Power Module specifications

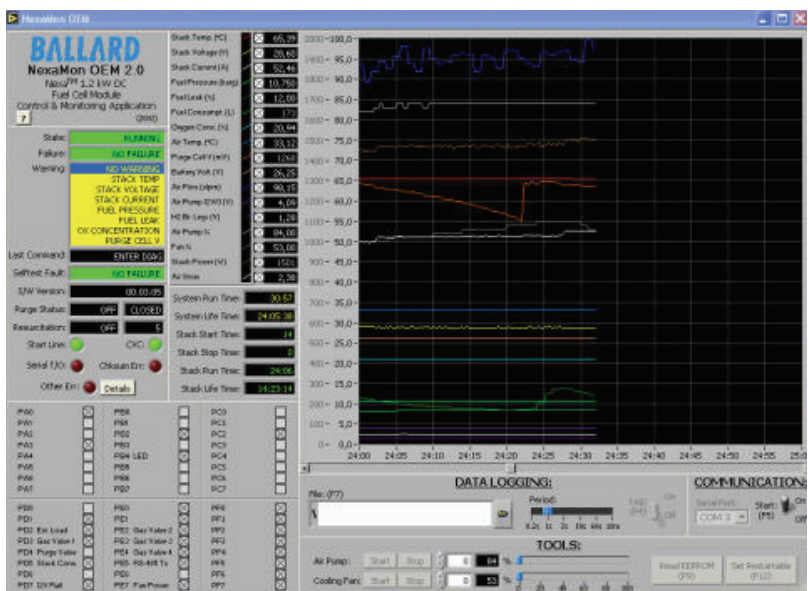

Fig. 11. Measured parameters in the fuel cell

As it has been justify in the previous section, for this fuel cell, the ANN structure which presents good results in the start up transient and the steady state is the structure MLP $5-5-2$, with activation functions L-P. It means that the selected ANN structure is a Multilayer Perceptron with 5 
input data (fuel cell temperature, hydrogen consumption, hydrogen pressure, oxygen flow and inlet air temperature) and 2 output data (voltage and current). Besides, the ANN structure has a hidden layer with 5 neurons and the activation functions are log-sigmoid and linear. Finally, a logarithmic scale has been applied to the input data, as shown in expression (3).

The network has been validated with 2004 measured samples. The average error is $0.0025 \%$ and the maximum error $0.02 \%$. Thus, the results have good accuracy for both, the steady state and the start up transient. Additionally, the time response is low and a second hidden layer is avoided, simplifying the network structure.

Figure 12 shows the voltage and current curves of the fuel cell system Nexa Ballard of $1.2 \mathrm{~kW}$. This figure shows the simulated values and the measured data, from the start up to the steady state. It can be appreciated that the results obtained with the selected ANN structure and the real data are almost superimposed.

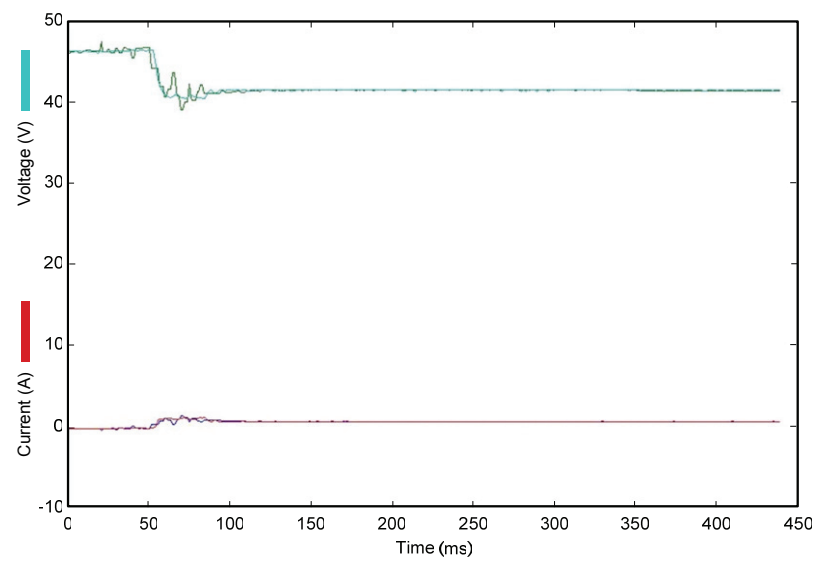

Fig. 12. PEMFC system voltage and current, in transient and steady state

\section{Conclusions}

Modelling of power generation systems, based on fuel cells, constitutes a fundamental challenge in the design of future electrical micro-grids and systems based on distributed generation. Conventional analytic models show difficulties in the identification of parameters because of the physical-chemical phenomena that are presented in these devices. Besides, these models are quite complex to be able to evaluate them with accuracy.

Therefore, once possible ways for characterising nonlinear systems have been analysed, this paper presents a model to simulate the behaviour of PEMFC systems, based on ANNs. The model allows predicting the behavior of the PEMFC technology in transient (start up) and steady state.

The training strategy and the ANN topology have been tested with a large number of parameters and network structures, to obtain high precision results. The software tool SARENEUR has been used in this process. The final ANN structure selected has been validated with experimental data obtained from the commercial device Nexa Ballard, of $1.2 \mathrm{~kW}$. Finally, it is interesting to point out that it has been necessary to normalize the input data using a logarithmic scale.

\section{Acknowledgements}

The work presented in this paper has been carried out by the research team of Project ENE2006-15700-CO2$02 / \mathrm{CON}$, supported by the Ministry of Education and Science of Spain and by the Regional Council of Guipuzcoa.

\section{References}

[1] M. Meiler, O. Schmid, M. Schudy, E.P. Hofer, "Dynamic fuel cell stack model for real-time simulation based on system identification", Journal of Power Sources, Vol.176, pp.523-528, 2008.

[2] R. Dittmar, B.M. Pfeiffer, "Modellbasierte prädiktive Regelung, Eine Einführung für Ingenieure", Oldenbourg Wissenschaftsverlag, München, Berlin, 2004.

[3] J.C. Amphlett, R.M. Baumert, R.F. Mann, B.A. Peppley, P.R. Roberge,T.J. Harris, "Performance Modeling of the Ballard Mark IV Solid Polymer Electrolyte Fuel Cell, I. Mechanistic Model", Journal of the Electrochem Society, Vol.142, pp.18, 1995.

[4] J.H. Lee, T.R. Lalk, "Modeling fuel cell stack systems", Journal of Power Sources, Vol.73, pp.229-241, 1998.

[5] W.Y. Lee, G.G. Park, T.H. Yang, Y.G. Yoon, H.S. Kim "Empirical modeling of polymer electrolyte membrane fuel cell performance using artificial neural networks", International Journal of Hydrogen Energy, Vol.29, pp.961-966, 2004.

[6] J.H. Lee, T.R. Lalk, A.J. Appleby, "Modeling electrochemical performance in large scale proton exchange membrane fuel cell stacks", Journal of Power Sources, Vol.70, pp.258-268, 1998.

[7] G. Squadrito, G. Maggio, E. Passalacqua, F. Lufrano, A. Patti, "An empirical equation for polymer electrolyte fuel cell behaviour", Journal Applied of the Electrochem Society, Vol.29, pp.1449-1455, 1999.

[8] S. Jemei, D. Hissel, M.C. Pera, J.M. Kauffmann, "On-board fuel cell power supply modeling on the basis of neural network methodology", Journal of Power Sources, Vol.124, pp.479-486, 2003.

[9] C.M. Bishop, "Neural Networks for Pattern Recognition”, Oxford University Press, 1995.

[10] S. Haykin, "Neural Networks: A Comprehensive. Foundation", $2^{\text {nd }}$ ed., Prentice-Hall, Inc., 1999.

[11] A.J. Mazon. I. Zamora. J. Gracia. K.J. Sagastebeitia. J.R. Saenz. "Selecting ANN structures to find transmission faults". IEEE Computer Applications in Power. Vol.14, n³, pp.44-48, July 2001. 